\title{
Intramammary lymph nodes
}

IH Jadusingh

\begin{abstract}
Aims: To determine the prevalence and pathology of intramammary lymph nodes in breast specimens.

Methods: All breast specimens examined by a single pathologist over 70 months in a large teaching hospital were studied retrospectively. All the surgical pathology reports were reviewed. Relevant glass slides from cases in which intramammary lymph nodes were identified were also reexamined.

Results: Breast specimens $(n=682)$ were examined. Seven lymph nodes were found in five patients. The specimens comprised 533 biopsy specimens, 29 segmental resections, 22 reduction mammoplasties, 77 modified radical mastectomies and 20 gynecomastia mastectomies. No clinically relevant microscopical abnormalities were found in four lymph nodes and slight sinus histiocytosis was seen in two nodes. One node contained metastatic adenocarcinoma and benign glandular epithelial inclusions.

Conclusion: Although rare, intramammary lymph nodes may be detected by careful gross examination of breast specimens even in the absence of clinical identification. They can occur in any quadrant of the breast and can display a variety of pathological conditions. Pathologists should be alert to the existence and potential importance of these lymph nodes.
\end{abstract}

( $\exists$ Clin Pathol 1992;45:1023-1026)

The occurrence of lymph nodes in the breast has not been extensively reported, even in anatomy textbooks. There is also a dearth of a single source of information on the pathological importance of these lymph nodes.

\section{Methods}

Department of Laboratory Medicine Calgary General Hospital, Calgary, Alberta, Canada IH Jadusingh

Correspondence to: Dr IH Jadusingh, Department of Laboratory Medicine, Calgary Genera Hospital-BVC, 841 Cente

Alberta T2E 0A1, Canada.

Accepted for publication 3 June 1992
The reports on all specimens of the breast in which the author performed a gross examination were reviewed over 70 months.

The hospital in which the cases were collected is a fairly large, university affiliated, teaching hospital with close to 1000 beds. The specimens were received mainly from general surgeons, with a smaller number from plastic surgeons, and the rare case from a general practitioner. Most patients had had surgery because of a clinically detected condition, but a smaller number were from patients in whom an abnormality had been detected by mammography.

As a component of the gross pathological examination, specimen mammography was performed on all cases in the following categories: (a) lesions detected by screening; (b) biopsy specimens taken after a preoperative mammographic needle localisation; (c) mammoplasty specimens in women aged over 30 years; (d) biopsy specimens measuring more than $3.0 \mathrm{~cm}$ in greatest dimensions, in which there was no grossly visible abnormality.

Gross examination was done in a routine manner with inspection of the external aspects and subsequent examination of thick slices (3-4 mm) of the entire specimen. Special clearance techniques by fat dissolution were not done. Whenever possible, the examination was done in conjunction with a review of the mammographic films. Selected sections were taken either for frozen sections, using a cryostat, or paraffin wax embedded sections. These were stained with haematoxylin and eosin and an occasional case was stained with special stains such as elastic van Gieson or periodic acid Schiff.

If the patient had more than one procedure done at the same time, the case was categorised by the larger specimen. A biopsy leading to immediate mastectomy was counted as a mastectomy specimen. Tylectomies were classified as biopsies. Patients with multiple procedures were counted as a single case.

To evaluate the specimen size and type of operation, the entire group was divided into biopsy, segmental mastectomy, reduction mammoplasty, modified radical mastectomy and mastectomy done for gynaecomastia specimens.

\section{Results}

A total of 681 cases were examined grossly. These comprised: 533 biopsy specimens, 29 segmental resections, 22 reduction mammoplasty specimens, 77 modified radical mastectomy and 20 gynaecomastia mastectomy specimens. The male:female ratio was about $1: 29$. The female patients ranged in age from 14 to 92 years with a mean of 44 years. Routine microscopical examination of paraffin wax embedded sections was done on all the cases and frozen section evaluation was done on 272 cases. Microscopical examination did not reveal any lymph nodes that were not already identified by gross evaluation.

Intramammary lymph nodes were found in specimens from five patients. Surgery was 
done because of a clinically palpable mass that was suspicious of malignancy in three patients and because of abnormal mammographic findings in two. None of the nodes had been palpated on clinical examination. Three patients had a modified radical mastectomy, one a segmental mastectomy, and the other a biopsy (tylectomy). Of the three modified radical mastectomies, two were done for infiltrating duct carcinoma and the other, on the youngest patient, was for a stromal sarcoma.

All the patients in whom intramammary lymph nodes were found were female. Their ages were $17,43,45,51$ and 75 years. The youngest (stromal sarcoma) and oldest (infiltrating duct carcinoma) each had two lymph nodes and a single node was found in each of

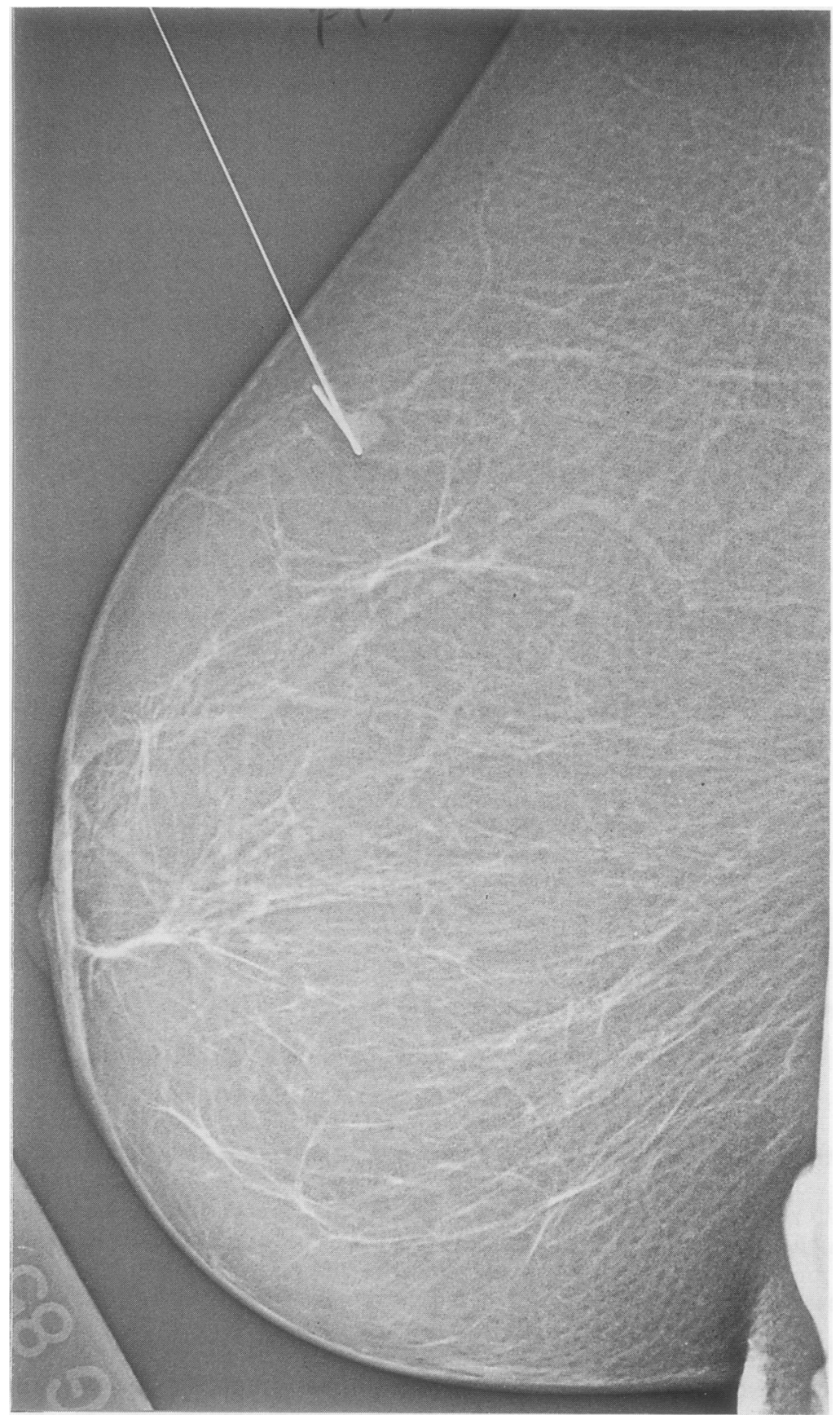

Figure 1 Preoperative fine wire localisation mammogram (nodule previously detected by a screening mammogram). Note small lymph node partially obscured by the tip of the wire.
Analysis of specimens

\begin{tabular}{lrl}
\hline Breast specimens & & $\begin{array}{l}\text { Intramammary } \\
\text { lymph nodes }\end{array}$ \\
\hline Biopsy specimens & 533 & 1 \\
Segmental resections & 29 & 1 \\
Reduction mammoplasties & 22 & \\
Modified radical mastectomies & 77 & 5 \\
Gynaecomastia mastectomies & 20 & 7 (5 patients) \\
Total & 681 & 7 . \\
\hline
\end{tabular}

\section{Discussion}

By definition, intramammary lymph nodes are surrounded by breast tissue and should be distinguished from low axillary lymph nodes. ${ }^{12}$ The fact that lymph nodes can be found in the breast is probably not generally appreciated and some standard textbooks of anatomy ${ }^{34}$ and surgical pathology 5 make no mention of them. The same deficiency occurs even in an article devoted exclusively to the anatomy and embryology of the breast. ${ }^{6}$ One popular book on the pathology of the breast does not mention the structures, ${ }^{7}$ but a more recent publication contains a short paragraph on the topic. "Haagenson describes "adventitious" and "pre-pectoral" nodes in his book on diseases of the breast. His subsequent discussion on their locations suggests that the nodes he is describing are not true intramammary lymph nodes. ${ }^{9}$ In contrast to this cursory coverage, published findings on radiology, particularly the publications of Egan have clearly defined these nodes. ${ }^{12}$ The nodes were 


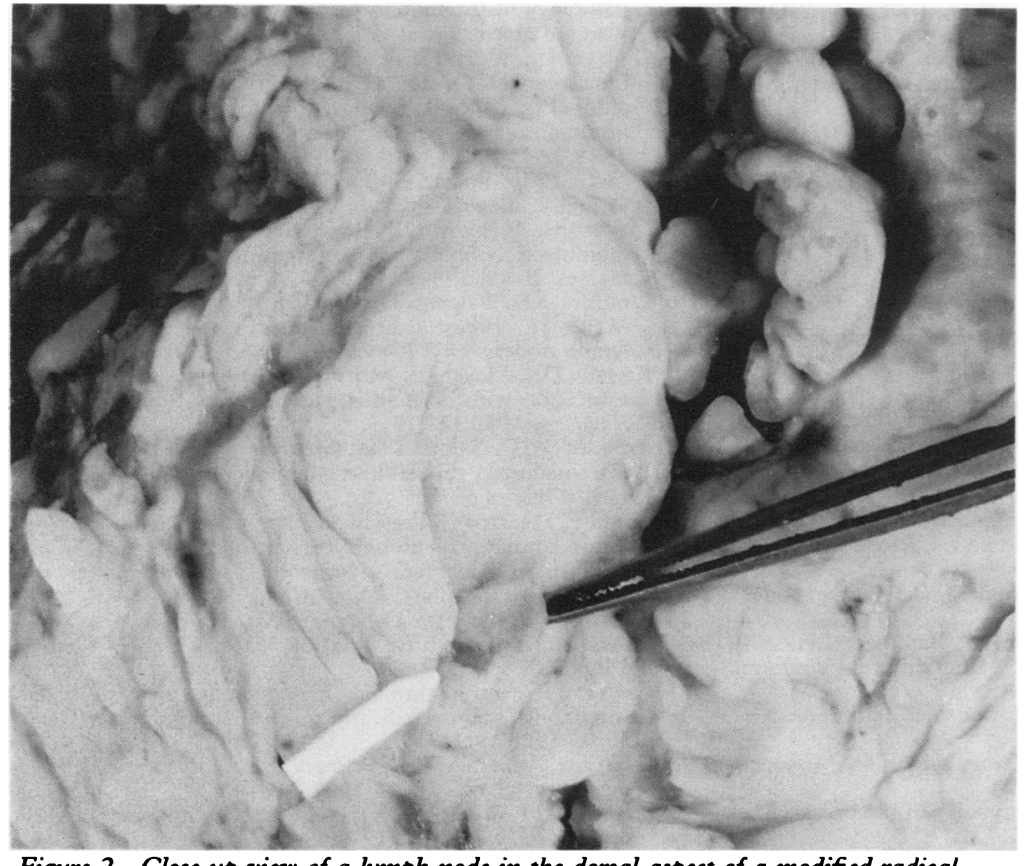

Figure 2 Close up view of a lymph node in the dorsal aspect of a modified radical mastectomy specimen (grasped by forceps, at the tip of the arrow).

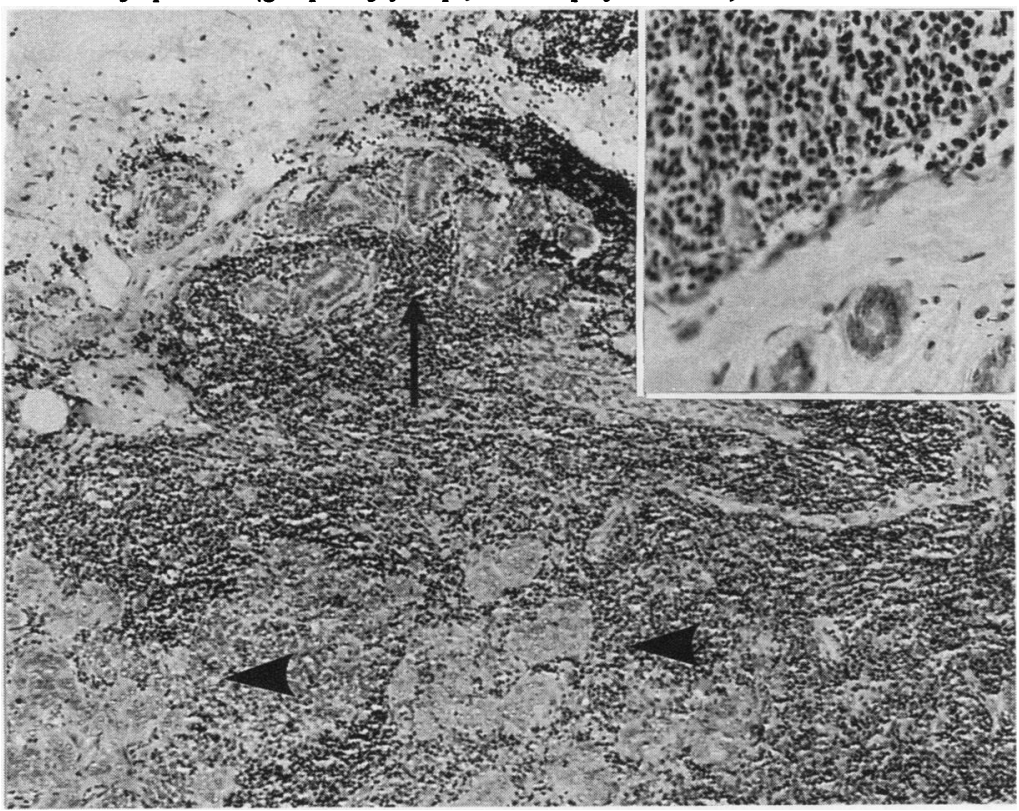

Figure 3 Intramammary lymph node with benign glandular inclusions (arrow) and metastatic adenocarcinoma (arrow heads). Inset: periphery of node with patent subcapsular sinus (haematoxylin and eosin).

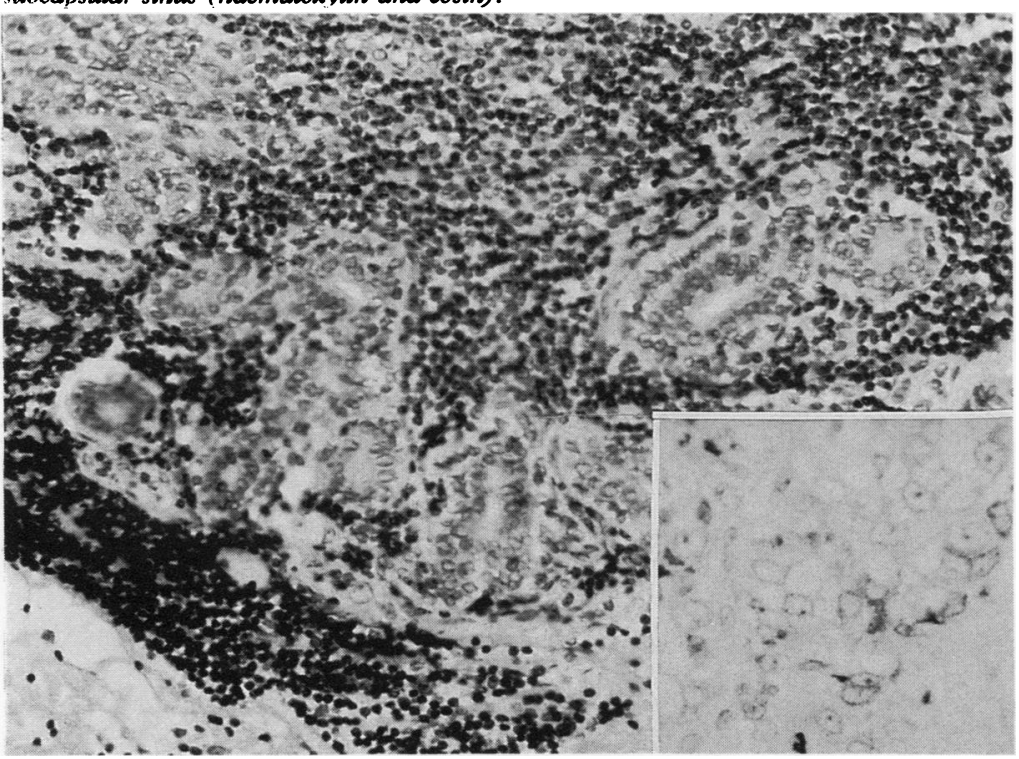

Figure 4 High power magnification of figure 3, showing benign glandular inclusions and metastatic carcinoma (inset) (haematoxylin and eosin). detected by radiographical examination of $5.0 \mathrm{~mm}$ thickness slices of "intact breast specimens". Surprisingly, pathological treatises in which the entire breast was examined do not mention these nodes, but it is difficult to tell if they were truly absent or if they were simply not mentioned. ${ }^{10-12}$

The prevalence is difficult to assess. Egan's showed the surprisingly high figure of $28 \%$ (45 of 158 breasts). Five cases of normal intramammary lymph nodes were detected in 1030 sonograms of palpable or mammographically detected breast masses by Gordon and Gilks. ${ }^{13}$ Eighteen patients with 19 lymph nodes presented with breast lumps over a six year period at the Charing Cross Hospital. ${ }^{14}$ In 108 excisional biopsy specimens of occult breast masses which were subjected to guided preoperative needle localisations, six normal intramammary lymph nodes were detected at the Massachusetts General Hospital. ${ }^{15}$ Three cases with metastatic carcinoma to intramammary lymph nodes occurring in 16000 mammograms were reported from the Massachusetts General Hospital. ${ }^{16} \mathrm{~A}$ total of seven nodes in five patients were found in the present study of 681 cases (see results section for case breakdown). An estimate of the prevalence of intramammary lymph nodes is difficult because of the variation in the methods of analysis in the aforementioned papers.

The reported sizes vary from $0.3 \mathrm{~cm}$ to $3.0 \mathrm{~cm}$ in diameter. Most seem to be about $1.0 \mathrm{~cm}$ in diameter. In most instances only a single node is identified with a propensity to be located in the upper outer quadrant, but as many as nine have been reported involving all quadrants of the breast. ${ }^{2}$

Most intramammary lymph nodes are probably detected by mammography and most will be normal. This paper represents one of the few reports in which the nodes were detected on the basis of gross pathological examination during routine surgical pathology in contrast to detection in more careful studies using specimen mammography of the entire breast. Pathological nodes may or may not be palpable. Pathological conditions that have been reported include metastatic carcinoma with a primary in the ipsilateral breast, ${ }^{12}$ carcinoma simulating medullary carcinoma without evidence of cancer in the mastectomy specimen or elsewhere, ${ }^{16}$ fibrosis, lymphoid hyperplasia, sinus histiocytosis, melanin pigmentation (which may or may not be associated with other features of dermatopathic lymphadenopathy $)^{17}$ fatty infiltration, a capsular naevus, ${ }^{14}$ tuberculosis, ${ }^{18}$ non-Hodgkin's malignant lymphoma ${ }^{1920}$ and gold deposition simulating microcalcifications on mammography in a patient who had received chrysotherapy for rheumatoid arthritis about 17 years earlier. ${ }^{21}$

Intramammary lymph nodes can be affected by any disease that can involve lymph nodes in other areas of the body. Doubtless, because their existence is difficult to detect, publications on topics such as malignant lymphomas of the breast and medullary carcinoma do not, with rare exceptions, ${ }^{20}$ discuss these nodes. ${ }^{22-28}$

Although previous publications suggest that 
these structures have been largely ignored by pathologists, they will probably receive increasing attention as mammography for breast cancer screening becomes more widely used. It is hoped that this publication will increase awareness and prompt further studies on the pathological importance of intramammary lymph nodes.

Financial assistance was provided by the Calgary General Hospital.

I thank my secretary, Helen Mason, for her invaluable assistance in preparing the manuscript and Dr F Alexander, director, Department of Laboratory Medicine for funding.

1 McSweeney MB, Egan RL. Prognosis of breast cancer related to intramammary lymph nodes. Recent Res Cancer Res $1984 ; 90: 166-72$.

2 Egan RL, McSweeney MB. Intramammary lymph nodes. Cancer 1983;51:1838-42.

3 Williams PL, Warwick R, Dyson M, Bannister LH, eds Gray's Anatomy. Edinburgh: Churchill Livingstone, 989.

4 Basmajian JV. Grant's Method of Anatomy. Baltimore: Williams \& Wilkins, 1980.

5 Rosai J. Ackerman's Surgical Pathology. St Louis, Missouri, CV Mosby Company, 1989.

6 Morehead JR. Anatomy and embryology of the breast. Clin Obstet Gynaecol 1982;25:353-7.

7 Azzopardi JG, Ahmed A, Millis RR. Major problems in pathology. Philadelphia: WB Saunders Company Ltd, 1979.

8 Page DL, Anderson TJ, eds. Diagnostic histopathology of the breast. Edinburgh: Churchill Livingstone, 1987

9 Haagensen CD. Diseases of the breast. Philadelphia: WB Haagensen CD. Diseases of
Saunders Company, 1986.

10 Jensen HM. On the origin and progression of human breast cancer. Obstet Gynecol 1986;154:1280-4.

11 Wellings SR, Jensen HM, Marcum RG. An atlas of subgross pathology of the human breast with special reference to possible precancerous lesions. FNCI 1975;55:231-73.

12 Gallager HS, Martin JE. Early phases in the development of breast cancer. Cancer 1969;24:1170-8.

13 Gordon PB, Gilks B. Sonographic appearance of normal intramammary lymph nodes. $f$ Ultrasound Med intramammary

14 Dawson PM, Shousha S, Burn JI. Lymph nodes presenting as breast lumps. Br $\mathcal{Y}$ Surg 1987;74:1167-8.

15 Meyer JE, Kopans DB, Lawrence WD. Normal intramammary lymph nodes presenting as occult breast masses. Breast 1982;8:30-2.

16 Lindfors KK, Kopans DB, McCarthy KA, Koerner FC, Meyer JE. Breast cancer metastasis to intramammary lymph nodes. Am F Rheum 1986;146:133-6.

17 Kopans DB, Mayer JE, Murphy GF. Benign lymph nodes associated with dermatitis presenting as breast masses. Radiology 1980;137:15-9.

18 Arnaout AH, Shousha S, Metaxas N, Husain OAN. Intramammary tuberculous lymphadenitis. Histopathology 1990;17:91-3.

19 Caimi F, Menozzi C, Freschi M. Localizzazione linfonodale intramammaria di linfoma malign non-Hodgkin (Non-
Hodgkin's malignant lymphoma in intramammary Hodgkin's malignant lymphoma in intramammar

20 Meis JM, Butler JJ, Osborne BM. Hodgkin's disease involving the breast and chest wall. Cancer 1986;57:1859-65.

21 Carter TR. Intramammary lymph node gold deposits simulating microcalcifications on mammogram. Hum Pathol 1988;19:992-4.

22 Jenstrom P, Sether JM. Primary lymphosarcoma of the mammary gland. $\mathfrak{f} A M A 1967 ; 201: 503-6$.

23 Schouten IT, Weese JL, Carbone PP. Lymphoma of the breast. Ann Surg 1981;194;749-53.

24 Telesinghe PU, Anthony PP. Primary lymphoma of breast. Histopathology 1985;9:297-307.

25 Wiseman C, Liao KT. Primary lymphoma of the breast. Cancer 1972;29:1705-12.

26 Jaffe ES. Surgical pathology of the lymph nodes and related organs. Major Prob Pathol 1985;16:298-9.
or

27 Ridolfi RL, Rosen PP, Port AP, Kinne D, MikeV, Medullary carcinoma of the breast. Cancer 1977;40:1365-85.

28 Mambo NC, Burke JS, Butler JJ. Primary malignan lymphomas of the breast. Cancer 1977;39:2033-40. 